\title{
Clinical outcomes of capecitabine-based versus S-1-based regimens as first-line chemotherapy in patients with unresectable or metastatic gastric cancer: a propensity score matched single- center comparison
}

\author{
Jin Wang ${ }^{1,2,3,4}$, Zhi Li ${ }^{1,2,3,4}$, Jinglei Qu ${ }^{1,2,3,4}$, Na Song ${ }^{1,2,3,4}$, Ying Chen ${ }^{1,2,3,4}$, Yu Cheng ${ }^{1,2,3,4}$, Simeng Zhang ${ }^{1,2,3,4}$, \\ Xiujuan $Q u^{1,2,3,4}$, Yunpeng Liu ${ }^{1,2,3,4}$ \\ ${ }^{1}$ Department of Medical Oncology, ${ }^{2}$ Key Laboratory of Anticancer Drugs and Biotherapy of Liaoning Province, ${ }^{3}$ Liaoning Province Clinical \\ Research Center for Cancer, ${ }^{4}$ Key Laboratory of Precision Diagnosis and Treatment of Gastrointestinal Tumors, Ministry of Education, the First \\ Hospital of China Medical University, Shenyang, China \\ Contributions: (I) Conception and design: J Wang, X Qu, Y Liu; (II) Administrative support: X Qu, Y Liu; (III) Provision of study materials or \\ patients: J Wang, Z Li, X Qu, Y Liu; (IV) Collection and assembly of data: J Qu, N Song, Y Chen, Y Cheng, S Zhang; (V) Data analysis and \\ interpretation: J Wang, Z Li; (VI) Manuscript writing: All authors; (VII) Final approval of manuscript: All authors. \\ Correspondence to: Xiujuan Qu; Yunpeng Liu. Department of Medical Oncology, the First Hospital of China Medical University, 155 Nanjing North \\ Street, Shenyang 110001, China. Email: xiujuanqu@yahoo.com; ypliu@cmu.edu.cn.
}

\begin{abstract}
Background: Fluoropyrimidine-based regimens are the cornerstone of first-line chemotherapy for metastatic gastric cancer (GC). Capecitabine or S-1 might be used as an alternative to infusional 5-fluorouracil, especially in pan-Asian. This study aimed to compare the clinical outcomes of capecitabine-based and S-1-based regimens as first-line chemotherapy in Chinese patients with unresectable or metastatic GC.

Methods: We conducted a retrospective study including unresectable or metastatic GC patients treated with the capecitabine-based or S-1-based regimen as first-line chemotherapy at the First Hospital of China Medical University. Propensity score matching (PSM) analysis was performed to reduce selection bias. Overall survival (OS) outcomes were compared using the Kaplan-Meier method and log-rank test. Prognostic significance was determined using multivariate Cox regression analysis. In addition, subgroup analyses were conducted to determine the effectiveness of capecitabine-based and S-1-based regimens in clinically relevant patient subsets.

Results: The clinical data of 469 patients included between October 2005 and September 2018. PSM analysis identified 187 patients receiving capecitabine-based or S-1-based regimen. No significant difference in OS (10.7 vs. 11.1 months, $\mathrm{P}=0.523)$ was detected between the two groups after PSM. In the subgroup analysis, the median OS (12.2 vs. 9.3 months, $\mathrm{P}=0.013)$ was longer for patients with peritoneum metastasis who received the capecitabine-based regimen compared to those who received the S-1-based regimen.

Conclusions: No significant difference in clinical outcomes was observed between the capecitabine and S-1-based regimen as first-line chemotherapy for metastatic or unresectable GC patients in China. The capecitabine-based regimen should be considered in the treatment of the GC patients with peritoneum metastasis.
\end{abstract}

Keywords: Gastric cancer (GC); first-line; chemotherapy; capecitabine; S-1

Submitted Feb 01, 2020. Accepted for publication Jun 12, 2020.

doi: 10.21037/jgo-20-52

View this article at: http://dx.doi.org/10.21037/jgo-20-52 


\section{Introduction}

Gastric cancer (GC) is one of the most common malignancies worldwide, and it shows highest annual incidence and mortality in China (1). In comparison with other countries, the initial diagnosis of GC is often delayed in China. Most patients are diagnosed at a late stage due to inadequate early diagnosis, particularly in rural and underdeveloped areas of China (2). Palliative chemotherapy is still a major medical treatment in metastatic or recurrent GC, however, there is no consensus as to the best firstline approach for unresectable or metastatic GC (3). It is necessary to further evaluate the clinical outcomes of various chemotherapy regimens for patients with unresectable or metastatic GC in the Chinese population.

Fluoropyrimidine-based regimens are widely used as reference regimens for first-line chemotherapy of metastatic GC in the NCCN guidelines (4). Currently, oral fluoropyrimidines such as capecitabine and $\mathrm{S}-1$ are increasingly used owing to their safety, convenience and the efficacy (5-7). Capecitabine or S-1 can be used as an alternative to infusional 5 -fluorouracil in doublet regimens in pan-Asian, adapted from the European Society for Medical Oncology clinical practice guidelines (8). However, the optimal regimens based on capecitabine or S-1 remain controversial. Some investigators have reported efficacy and clinical outcomes of capecitabine and S- 1 in metastatic GC. In two phase II trials in advanced elderly patients with metastatic GC using capecitabine or S-1, the results showed that the median time-to-progression (TTP) and overall survival (OS) were similar in two groups $(9,10)$. As a doublet regimen of advanced GC, SOX (S-1 plus oxaliplatin) and CAPOX (capecitabine plus oxaliplatin) regimens showed no statistical difference in TTP (6.2 vs. 7.2 months, $\mathrm{P}=0.767)$ and $\mathrm{OS}$ (12.4 vs. 13.3 months, $\mathrm{P}=0.686)$ (11). The results of the phase II trial (XParTS II) indicated that SP (S-1 plus cisplatin) and XP (capecitabine plus cisplatin) regimens had no statistical difference in TTP [hazard ratio $(\mathrm{HR})=1.126$, $\mathrm{P}=0.563]$ and $\mathrm{OS}(\mathrm{HR}=0.942, \mathrm{P}=0.776)(12)$. Another phase II trial (OGSG1105, HERBIS-4A) compared the efficacy of $\mathrm{SP}$ and XP regimens in human epidermal growth receptor 2 (HER2)-negative AGC, showing no statistical difference in TTP (5.9 vs. 4.1 months, $\mathrm{P}=0.284)$ and $\mathrm{OS}$ (3.5 vs. 10.0 months, $\mathrm{P}=0.290$ ) (13). The efficacy of capecitabinebased and S-1 based chemotherapy regimens in the treatment of advanced GC still warrants further research.

This study was designed to investigate the differential clinical outcomes of capecitabine-based and S-1-based regimens as first-line chemotherapy for Chinese patients with metastatic or unresectable GC. Subgroup analyses were conducted on patients who are mostly likely to benefit from the different treatments. We present the following article in accordance with the STROBE reporting checklist (available at http://dx.doi.org/10.21037/jgo-20-52).

\section{Methods}

\section{Study population}

The clinical data of patients with metastatic or unresectable GC, who received capecitabine-based or S-1-based regimen as first-line chemotherapy at the First Hospital of China Medical University between October 2005 and September 2018, were collected.

The eligibility criteria of patients included: (I) aged $\geq 18$ years; (II) histologically confirmed diagnosis of GC; (III) at least one measurable or evaluable lesion; (IV) patients who received at least one cycle of chemotherapy; (V) availability of clinic-pathological data at the start of chemotherapy.

The exclusion criteria were as follows: (I) esophageal cancer, squamous cell carcinomas, or gastroesophageal junction tumors; (II) any prior palliative chemotherapy or radiotherapy for malignancy within the past 5 years; (III) adjuvant or neo-adjuvant therapy within the past 12 months.

\section{Chemotherapy regimen}

Patients received either capecitabine-based or S-1based regimen according to physician preference. In the capecitabine-based regimen, capecitabine was provided at $1,000 \mathrm{mg} / \mathrm{m}^{2}$ in combination regimens, $1,500 \mathrm{mg} / \mathrm{m}^{2}$ in single regimen, twice a day on days $1-14$. The S-1-based regimen was planned as $40 \mathrm{mg} / \mathrm{m}^{2}$ orally administered twice a day on days 1-14. The combination regimens contained oxaliplatin, cisplatin, paclitaxel and docetaxel, and relative dose intensities of the partner drugs were similar in the two groups. The two regimens were repeated every three weeks.

\section{Data collection}

Using the medical records of the patients, the following information was collected, including demographics, Eastern Cooperative Oncology Group performance status (ECOG PS), history of previous surgery, tumor location, 
tumor differentiation, HER2 expression status, distant metastatic status and chemotherapeutic regimens. Patients were followed up every three months. The main endpoint was OS, defined as the period from the unresectable or metastatic time to death or the last follow-up visit, which was December 15, 2018.

\section{Propensity score matching (PSM)}

PSM analysis, which matched multiple factors at the same time and maximized the reduction of confounding bias $(14,15)$, was performed to correct for bias between the effects of two regimens groups. The propensity score was calculated using multivariable logistic regression for each patient. The propensity score model included the following baseline covariates: age, gender, ECOG PS, history of previous surgery, tumor location, tumor differentiation, the number of distant metastatic sites, as well as the metastasis status of the bone, liver, lung and peritoneum. Then we matched participants using a simple 1:1 nearest neighbor matching, leading to an even distribution of potential confounding factors in the two groups. The maximum caliper width was 0.05 of the propensity score difference.

\section{Statistical analysis}

Categorical variables were presented as numbers and percentages. Continuous variables were presented as the mean \pm SD. The clinic-pathological parameters between two groups were analyzed by using a Chi-square test, or Fisher's exact test in the observational and the PSM datasets. The standardized mean difference (SMD) in each variable was calculated to determine whether the PSM was adequately conducted. An SMD more than 0.1 was considered to represent meaningful covariate imbalance (16). Kaplan-Meier curves with the log-rank tests were adopted to calculate the overall cumulative probability. Cox regression analyses were then used to evaluate independent prognostic factors. Variables that achieved a significant level of $\mathrm{P}<0.2$ were conducted the multivariate analyses. $\mathrm{P}$ values of $<0.05$ were considered to be statistically significant. All $\mathrm{P}$ values corresponded to two-sided significance tests. Statistical analyses were performed using SPSS 23.0 software package (SPSS, Chicago, IL) and R software, version 3.3.1 (http://www.r-project.org).

The study was conducted in accordance with the Declaration of Helsinki (as revised in 2013). The study protocol was approved by the Ethics Committee of the
First Hospital of China Medical University (No. AFSOP-07-1.1-01) and written informed consent was obtained from all patients.

\section{Results}

\section{Patient characteristics}

From October 2005 to September 2018, the clinical data of 469 metastatic or unresectable GC patients were included in this study. Of these patients, 195 received a capecitabinebased regimen and 274 patients received an S-1-based regimen as first-line chemotherapy. The most commonly used chemotherapeutic type of regimen was oxaliplatincombined regimen, CAPOX $(\mathrm{n}=79,40.5 \%)$ and SOX $(\mathrm{n}=122,44.5 \%)$ regimen, respectively. The next was taxanecombined (paclitaxel or docetaxel) regimen, $26.7 \%$ in capecitabine group and $20.8 \%$ in S-1 group, respectively.

In the observational dataset, there was a significant difference between the two groups in ECOG PS $(\mathrm{P}=0.004)$, the status of peritoneum metastasis $(\mathrm{P}=0.008)$ and liver metastasis $(\mathrm{P}=0.015)$. After PSM at ratio of $1: 1$, a wellbalanced cohort of 374 patients remained in the analysis. There were no significant differences among potential prognostic factors observed in these two groups $(\mathrm{P}>0.05$ for all). The baseline characteristics of the patients before and after PSM were listed in Table 1.

\section{Survival outcomes}

The median time of follow-up was 52 months in both the observational and PSM datasets. In the observational dataset, there was no significant difference in OS (10.7 vs. 11.0 months, $\mathrm{P}=0.950$ ) between the two groups (Figure $1 A$ ). In multivariate analysis, the history of previous gastrectomy, number of distant metastasis sites and bone metastasis were the independent factors (Table S1). In the head-tohead PSM analysis, application of the capecitabine group resulted in similar OS to that observed with S-1 group (10.7 vs. 11.1 months, $\mathrm{P}=0.523$, Figure $1 B$ ). The results of the multivariate analysis indicated that the history of previous gastrectomy (HR $=0.617,95 \%$ CI, 0.493-0.772) and number of distant metastasis sites (HR $=1.283,95 \% \mathrm{CI}$, 1.126-1.462) were the independent factors (Table 2).

\section{Subgroup analysis}

In the further subgroup analysis of the PSM dataset, there 
Table 1 Comparison of characteristics in the observational and propensity score matching datasets

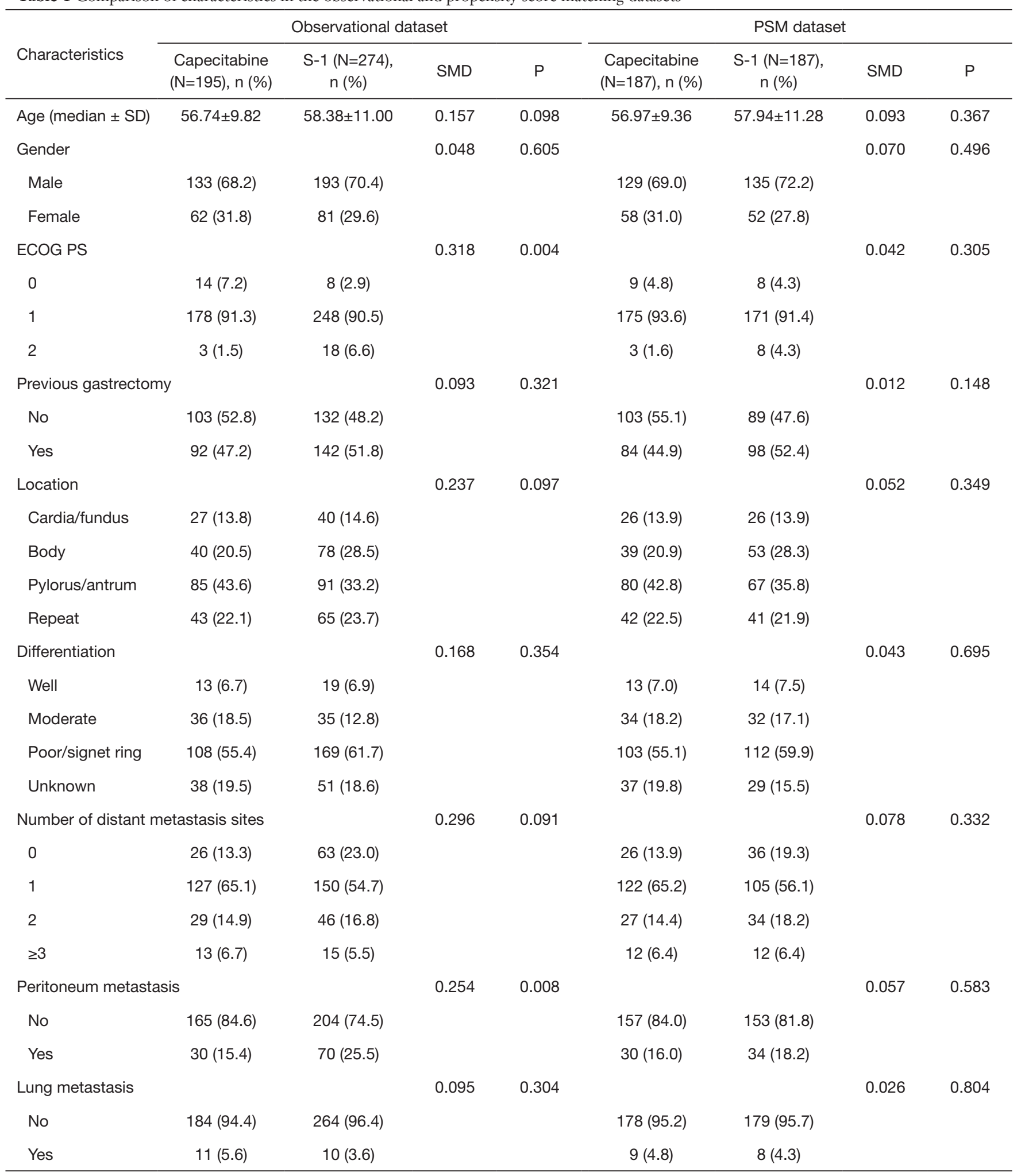

Table 1 (continued) 
Table 1 (continued)

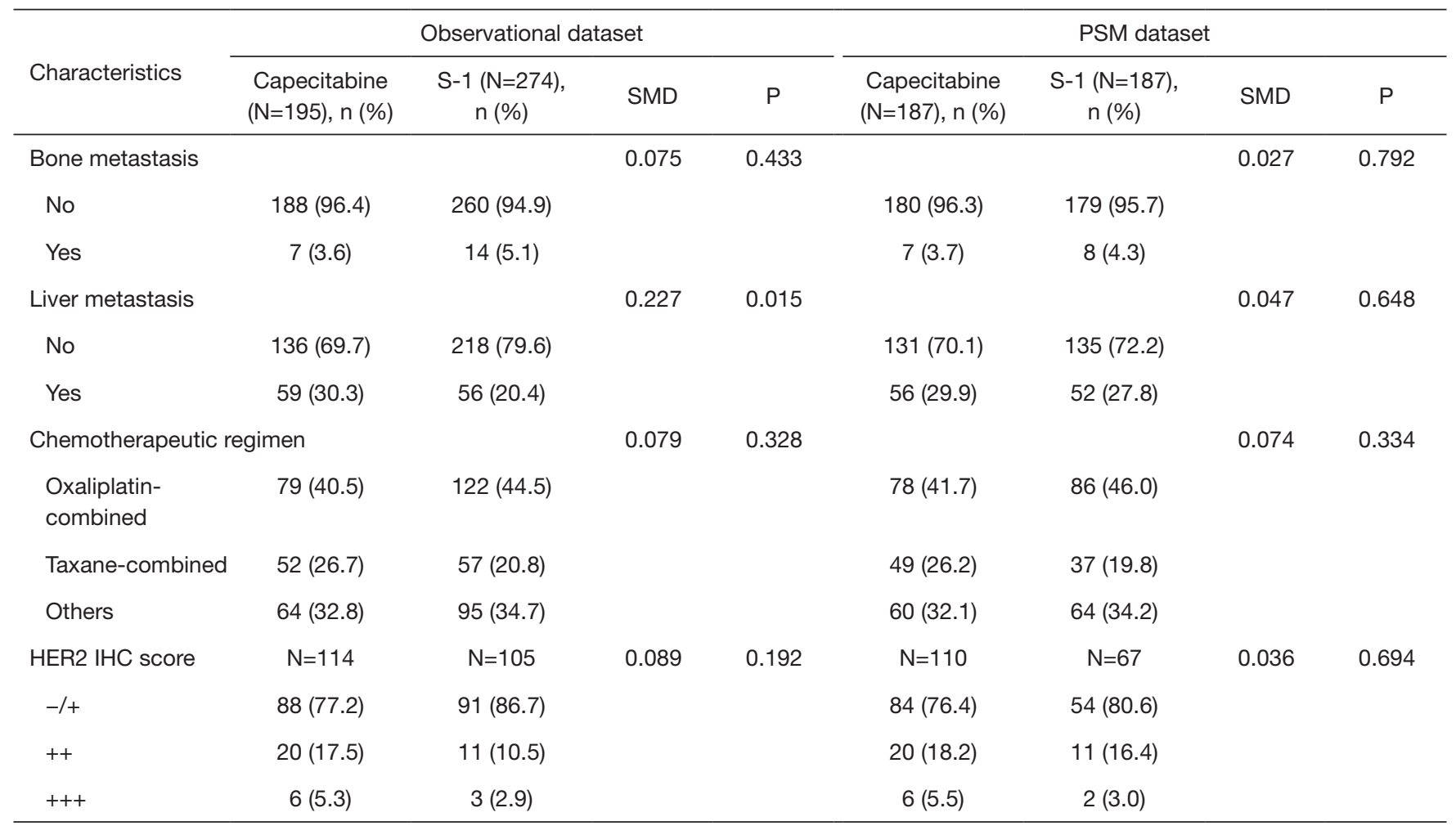

SMD, standardized mean difference; PSM, propensity score matching; SD, standard deviation; ECOG PS, Eastern Cooperative Oncology Group performance status; HER2, human epidermal growth factor receptor 2; IHC, immunohistochemistry.
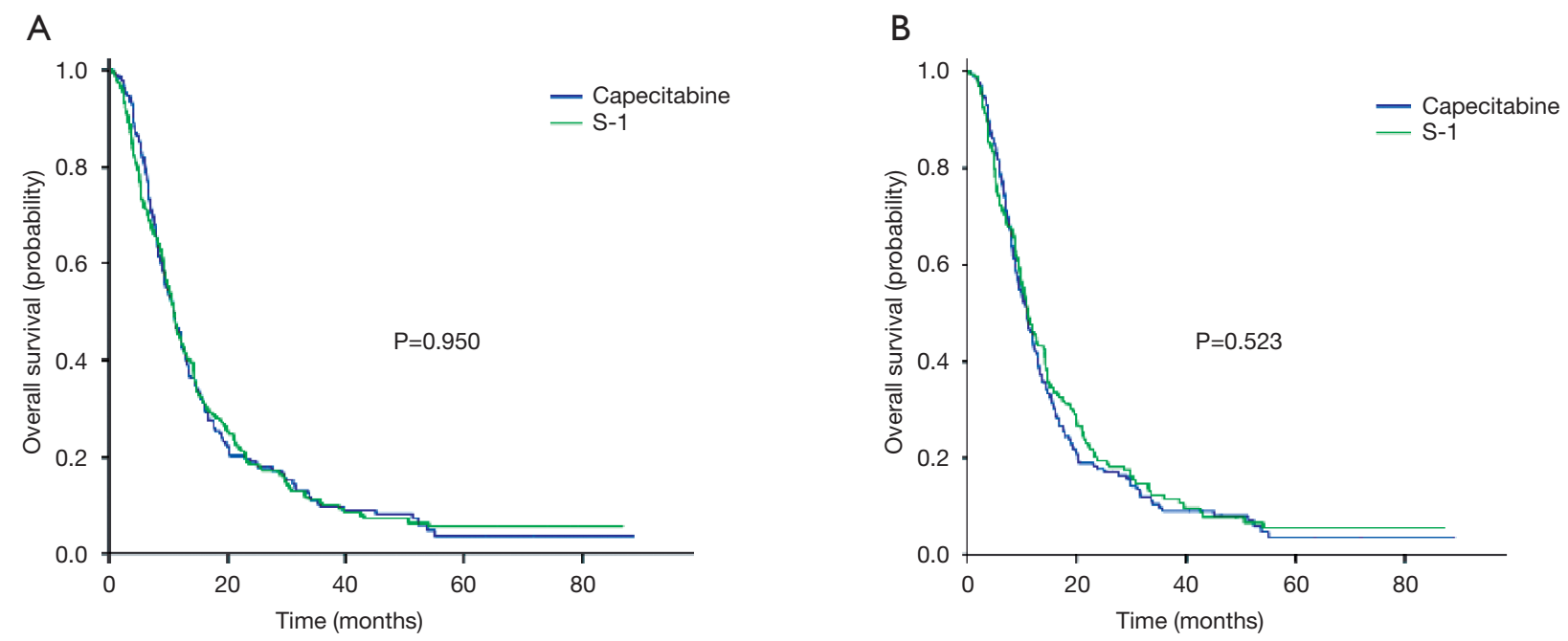

Figure 1 Overall survival plot in (A) the observational dataset, (B) propensity score matching analysis.

were no significantly different effects between two groups in OS, except for the peritoneum metastasis subgroup (Figure 2). In the peritoneum metastasis subgroup, patients who received the capecitabine-based regimen showed a better OS than those who received the S-1-based regimen (12.2 vs. 9.3 months, $\mathrm{P}=0.013$ ). Similar results were obtained 
Table 2 Univariate and multivariate analysis of clinical characteristics in relation to overall survival in PSM database

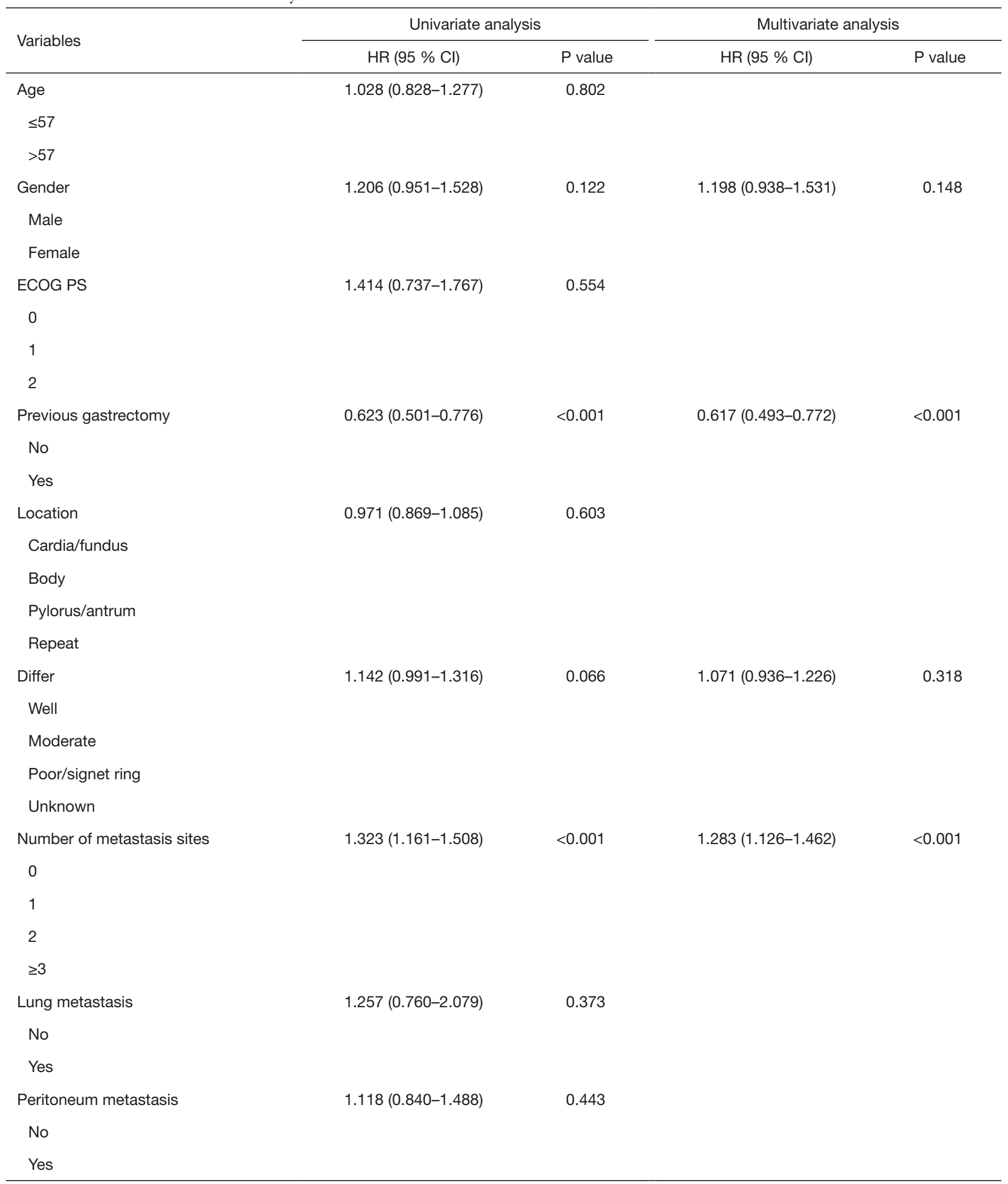

Table 2 (continued) 
Table 2 (continued)

\begin{tabular}{|c|c|c|c|c|}
\hline Variables & \multicolumn{2}{|c|}{ Univariate analysis } & \multicolumn{2}{|c|}{ Multivariate analysis } \\
\hline Liver metastasis & $1.256(0.988-1.595)$ & 0.062 & $1.137(0.881-1.469)$ & 0.324 \\
\hline \multicolumn{5}{|l|}{ No } \\
\hline \multicolumn{5}{|l|}{ Yes } \\
\hline \multicolumn{5}{|l|}{ No } \\
\hline Yes & & & & \\
\hline
\end{tabular}

PSM, propensity score matching; HR, hazard ratio; Cl, confidence interval; ECOG PS, Eastern Cooperative Oncology Group performance status.

\begin{tabular}{|c|c|}
\hline Variables & $\mathrm{N}$ \\
\hline \multicolumn{2}{|l|}{ Age } \\
\hline$\leq 57$ & 205 \\
\hline$>57$ & 169 \\
\hline \multicolumn{2}{|l|}{ Gender } \\
\hline Female & 110 \\
\hline Male & 264 \\
\hline \multicolumn{2}{|l|}{ Previous gastrectomy } \\
\hline No & 192 \\
\hline Yes & 182 \\
\hline \multicolumn{2}{|l|}{ ECOG PS } \\
\hline 0 & 17 \\
\hline 1 & 346 \\
\hline 2 & 11 \\
\hline \multicolumn{2}{|c|}{ Number of metastasis sites } \\
\hline 0 & 62 \\
\hline 1 & 227 \\
\hline 2 & 61 \\
\hline$\geq 3$ & 24 \\
\hline \multicolumn{2}{|l|}{ Bone metastasis } \\
\hline No & 359 \\
\hline Yes & 15 \\
\hline \multicolumn{2}{|l|}{ Liver metastasis } \\
\hline No & 266 \\
\hline Yes & 108 \\
\hline \multicolumn{2}{|l|}{ Peritoneum metastasis } \\
\hline No & 310 \\
\hline Yes & 64 \\
\hline \multicolumn{2}{|l|}{ Lung metastasis } \\
\hline No & 357 \\
\hline Yes & 17 \\
\hline \multicolumn{2}{|l|}{ Regimen } \\
\hline Oxaliplatin-combined & 164 \\
\hline Taxane combined & 86 \\
\hline Others & 124 \\
\hline
\end{tabular}

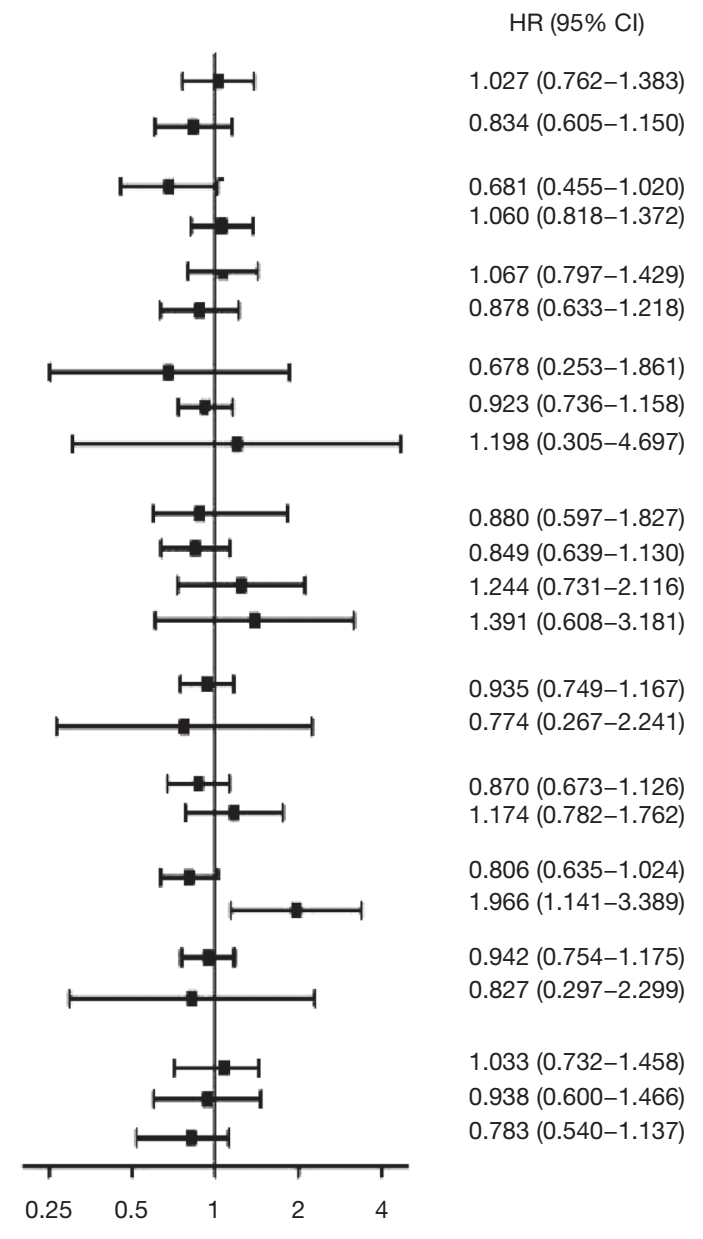

Favours S-1 Favours capecitabine

Figure 2 Forest plot of the treatment effects on overall survival in the subgroup analysis. 

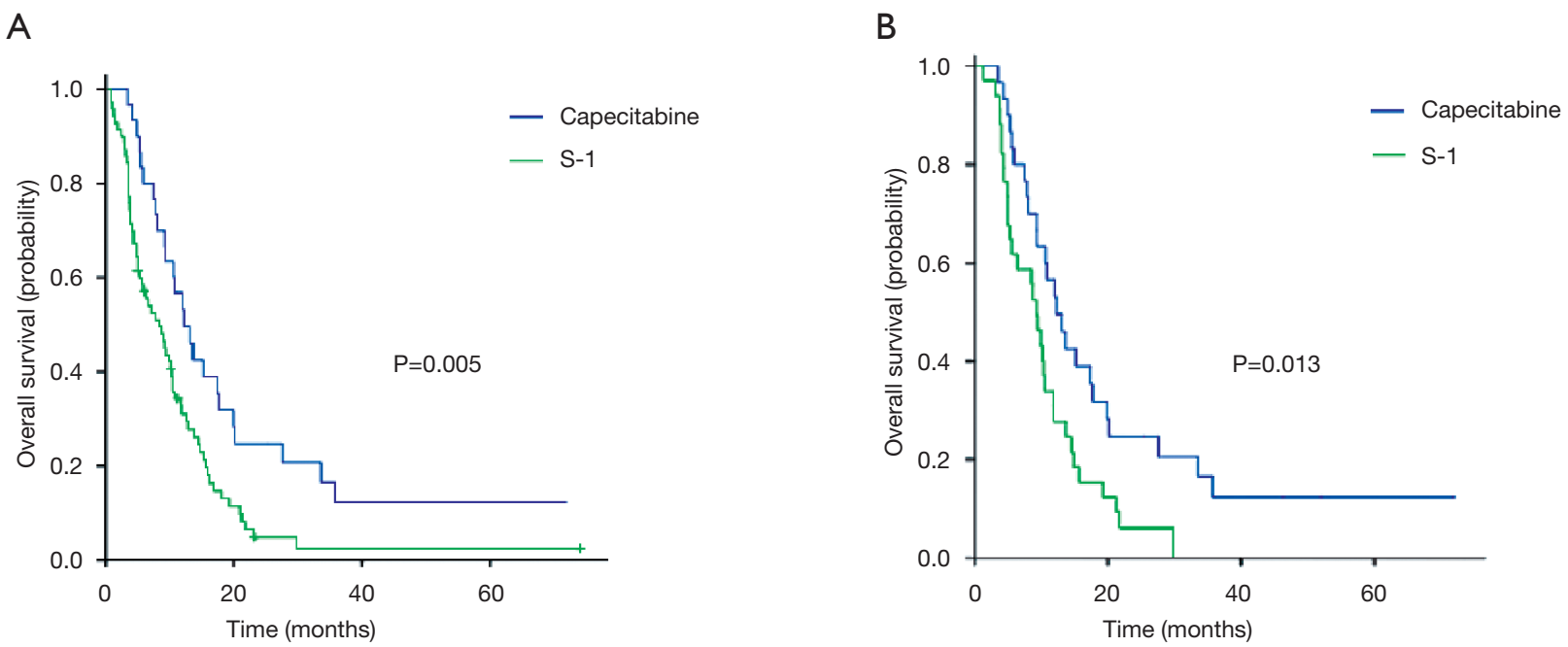

Figure 3 In the peritoneal metastasis subgroup, overall survival plot in (A) the observational dataset, (B) propensity score matching analysis.

in the observational dataset (12.2 months for capecitabine vs. 8.5 months for $\mathrm{S}-1, \mathrm{P}=0.005)$, shown in Figure 3. The patients who received the $\mathrm{S}-1$-based regimen showed a survival benefit trend in the female subgroup $(\mathrm{P}=0.062$; HR $=0.681,95 \%$ CI, $0.455-1.020)$ and the non-peritoneum metastasis subgroup $(\mathrm{P}=0.078$; HR $=0.806$, 95\% CI, 0.635 1.024).

\section{Discussion}

The positive impact of palliative chemotherapy on the survival of unresectable or metastatic patients with gastric adenocarcinoma has become obvious over time, although the best chemotherapy regimen has not yet been established. The capecitabine-based regimen has been widely used based on the REAL-2 and ML17032 trials (6,7). It is also frequently designed as the active comparator in phase III clinical trials to evaluate the potential of molecular targeted therapeutics for advanced GC (17-19). Conversely, an S-1-based regimen is the accepted standard treatment regimen for patients with advanced GC in Japan, according to the JCOG9912 and SPIRITS trials $(20,21)$. To the best of our knowledge, a capecitabine-based regimen has not been retrospectively compared with a S-1-based regimen as first-line chemotherapy of patients with metastatic or unresectable GC in China.

Our study showed that there was no significant difference in OS between two chemotherapy regimens. Our data are consistent with two previous meta-analyses which showed similar prognostic outcomes for the both regimens
$(22,23)$. Zhu et al. showed capecitabine and S-1 exhibited a significant OS benefit over 5-Fu in Asian population, with HRs of 0.88 (95\% CI, 0.80-0.96) and 0.90 (95\% CI, 0.810.99), respectively. Additionally, these regimens have similar results in terms of overall response rate and grade 3 or 4 adverse events (23). Thus it can be seen that capecitabine and S-1 might be competitive and interchangeable, especially in Asian.

In the exploratory subgroup analysis, we found that the capecitabine group had better OS $(\mathrm{P}=0.013)$ in the peritoneum metastasis subgroup and the $\mathrm{S}-1$ group had a trend towards better $\mathrm{OS}(\mathrm{P}=0.078)$ in the non-peritoneum metastasis subgroup. Based on current studies, it is still inconclusive whether capecitabine or S-1 should be used in GC patients with peritoneal metastasis. In recent years, some studies have achieved promising results, using these two drugs in combination with paclitaxel or oxaliplatin to treat GC with peritoneal metastasis (24-26). A multicenter phase III clinical trial in Japan (PHOENIX-GC) showed the median OS of intraperitoneal paclitaxel plus systemic S-1 and paclitaxel compared to SP for GC patients with peritoneal metastasis, showing OS of 17.7 and 15.2 months, respectively (24). The result of the phase II clinical trial of Singapore (NCT01739894), suggested that the median OS of intraperitoneal paclitaxel combined with systemic CAPOX for GC patients with peritoneal metastasis was 18.8 months, with a 1 -year survival rate of $72.2 \%$ (25). In another phase II trial in Korea (NCT01739894), the median OS of intraperitoneal docetaxel plus capecitabine and cisplatin for GC patients with peritoneal metastasis 
was 15.1 months (26). A phase II clinical trial in the United States (NCT04034251) that uses intraperitoneal paclitaxel and intravenous paclitaxel plus capecitabine is still on going. To date, there has been no head-to-head study of capecitabine versus S-1 in the treatment of GC with peritoneal metastasis. The results of the present study could enrich the theoretical basis, but further in-depth demonstration was needed.

Previous studies have shown that thymidine phosphorylase expression levels are predictors of the efficacy of capecitabine chemotherapy regimen, whilst thymidine phosphorylase is highly expressed in GC with liver metastases $(27,28)$. However, the efficacy of the capecitabine regimen in liver metastasis of GC remains inconclusive. The present study only analyzed the long-term survival outcomes, but did not investigate therapeutic effect. The capecitabine group showed extended OS compared to the S-1 group in the liver metastasis subgroup, which was not statistically significant. Therefore, more clinical studies are required to further verify these findings.

The limitations of this study must be acknowledged. Firstly, it is a retrospective study in a single institution. The patients received either the capecitabine-based or S-1based regimen according to physician preference, which corresponded to a non-randomized, unblinded setting with a high risk of selection bias. PSM analysis used in this study could balance the selection bias to some extent. The results are needed to be validated in multicenter, prospective cohort study. Secondly, the information about HER2 status was incomplete, approximately $40 \%$ missing values. Because the patients enrolled in present study were from 2005, and the test of HER2 status for metastatic advanced GC was not a routine examination in early years after ToGA (Trastuzumab for Gastric Cancer) study reported (17). After 2014, the detection rate of HER2 can reach close to $70 \%$ (data not shown). Considering HER2 status was not concerned with capecitabine or S-1 medication, this study did not include HER2 status into the propensity-score model. Thirdly, this study did not provide toxicity and response analysis results by considering the relatively clear toxicity of these two drugs. Fourthly, the lack of comparison of progression-free survival and subsequent treatment between the two groups has brought limitations to a more in-depth discussion.

\section{Conclusions}

In conclusion, our study suggested that there was no significant difference in OS between capecitabine-based and
S-1-based regimen as first-line chemotherapy of patients with metastatic or unresectable GC in China. We find that the use of the capecitabine-based regimen should be taken into consideration in the treatment of GC patients with peritoneum metastasis. Further large-scale randomized studies focusing on certain types of regimen are needed to validate the optimal treatment strategy.

\section{Acknowledgments}

Funding: This research was funded by National Science and Technology Major Project of the Ministry of Science and Technology of China (No. 2017ZX09304025); the National Natural Science Foundation of China (No. 81902998); Science and Technology Plan Project of Liaoning Province (No. 2016007010); The General Projects of Liaoning Province Colleges and Universities (No. LFWK201706); Science and Technology Youth Projects of the Education Department of Liaoning Province (QN2019004).

\section{Footnote}

Reporting Checklist: The authors have completed the STROBE reporting checklist. Available at http://dx.doi. org/10.21037/jgo-20-52

Conflicts of Interest: All authors have completed the ICMJE uniform disclosure form (available at http://dx.doi. org/10.21037/jgo-20-52). The authors have no conflicts of interest to declare.

Ethical Statement: The authors are accountable for all aspects of the work in ensuring that questions related to the accuracy or integrity of any part of the work are appropriately investigated and resolved. The study was conducted in accordance with the Declaration of Helsinki (as revised in 2013). The study protocol was approved by the Ethics Committee of the First Hospital of China Medical University (No. AF-SOP-07-1.1-01) and written informed consent was obtained from all patients.

Open Access Statement: This is an Open Access article distributed in accordance with the Creative Commons Attribution-NonCommercial-NoDerivs 4.0 International License (CC BY-NC-ND 4.0), which permits the noncommercial replication and distribution of the article with the strict proviso that no changes or edits are made and the original work is properly cited (including links to both the 
formal publication through the relevant DOI and the license). See: https://creativecommons.org/licenses/by-nc-nd/4.0/.

\section{References}

1. Chen W, Zheng R, Baade PD, et al. Cancer statistics in China, 2015. CA Cancer J Clin 2016;66:115-32.

2. Nie $\mathrm{Y}, \mathrm{Wu} \mathrm{K}, \mathrm{Yu} \mathrm{J}$, et al. A global burden of gastric cancer: the major impact of China. Expert Rev Gastroenterol Hepatol 2017;11:651-61.

3. Orditura M, Galizia G, Sforza V, et al. Treatment of gastric cancer. World J Gastroenterol 2014;20:1635-49.

4. National Comprehensive Cancer Network. Gastric cancer (version 2.2019). Available online: https://www.nccn.org/ professionals/physician_gls/pdf/gastric.pdf. Accessed Jun 3, 2019.

5. Ajani JA, Rodriguez W, Bodoky G, et al. Multicenter phase III comparison of cisplatin/S-1 with cisplatin/infusional fluorouracil in advanced gastric or gastroesophageal adenocarcinoma study: the FLAGS trial. J Clin Oncol 2010;28:1547-53.

6. Cunningham D, Starling N, Rao S, et al. Capecitabine and oxaliplatin for advanced esophagogastric cancer. N Engl J Med 2008;358:36-46.

7. Kang YK, Kang WK, Shin DB, et al. Capecitabine/ cisplatin versus 5 -fluorouracil/cisplatin as first-line therapy in patients with advanced gastric cancer: a randomised phase III noninferiority trial. Ann Oncol 2009;20:666-73.

8. Muro K, Van Cutsem E, Narita Y, et al. Pan-Asian adapted ESMO Clinical Practice Guidelines for the management of patients with metastatic gastric cancer: a JSMO-ESMO initiative endorsed by CSCO, KSMO, MOS, SSO and TOS. Ann Oncol 2019;30:19-33.

9. Lee JL, Kang YK, Kang HJ, et al. A randomised multicentre phase II trial of capecitabine vs S-1 as first-line treatment in elderly patients with metastatic or recurrent unresectable gastric cancer. Br J Cancer 2008;99:584-90.

10. Kim MJ, Kong SY, Nam BH, et al. A randomized phase II study of S-1 versus capecitabine as first-line chemotherapy in elderly metastatic gastric cancer patients with or without poor performance status: clinical and pharmacogenetic results. Pharmacogenet Genomics 2018;28:23-30.

11. Kim GM, Jeung HC, Rha SY, et al. A randomized phase II trial of S-1-oxaliplatin versus capecitabine-oxaliplatin in advanced gastric cancer. Eur J Cancer 2012;48:518-26.

12. Nishikawa K, Tsuburaya A, Yoshikawa T, et al. A randomised phase II trial of capecitabine plus cisplatin versus S-1 plus cisplatin as a first-line treatment for advanced gastric cancer: Capecitabine plus cisplatin ascertainment versus S-1 plus cisplatin randomised PII trial (XParTS II). Eur J Cancer 2018;101:220-8.

13. Kawakami H, Takeno A, Endo S, et al. Randomized, Open-Label Phase II Study Comparing CapecitabineCisplatin Every 3 Weeks with S-1-Cisplatin Every 5 Weeks in Chemotherapy-Naive Patients with HER2-Negative Advanced Gastric Cancer: OGSG1105, HERBIS-4A Trial. Oncologist 2018;23:1411-e147.

14. D'Agostino RB Jr. Propensity score methods for bias reduction in the comparison of a treatment to a nonrandomized control group. Stat Med 1998;17:2265-81.

15. Hwang ES, Wang X. Value of Propensity Score Matching to Study Surgical Outcomes. Ann Surg 2017;265:457-8.

16. Austin PC. An Introduction to Propensity Score Methods for Reducing the Effects of Confounding in Observational Studies. Multivariate Behav Res 2011;46:399-424.

17. Bang YJ, Van Cutsem E, Feyereislova A, et al. Trastuzumab in combination with chemotherapy versus chemotherapy alone for treatment of HER2-positive advanced gastric or gastro-oesophageal junction cancer (ToGA): a phase 3, open-label, randomised controlled trial. Lancet 2010;376:687-97.

18. Ohtsu A, Shah MA, Van Cutsem E, et al. Bevacizumab in combination with chemotherapy as first-line therapy in advanced gastric cancer: a randomized, doubleblind, placebo-controlled phase III study. J Clin Oncol 2011;29:3968-76.

19. Lordick F, Kang YK, Chung HC, et al. Capecitabine and cisplatin with or without cetuximab for patients with previously untreated advanced gastric cancer (EXPAND): a randomised, open-label phase 3 trial. Lancet Oncol 2013;14:490-9.

20. Boku N, Yamamoto S, Fukuda H, et al. Fluorouracil versus combination of irinotecan plus cisplatin versus $\mathrm{S}-1$ in metastatic gastric cancer: a randomised phase 3 study. Lancet Oncol 2009;10:1063-9.

21. Koizumi W, Narahara H, Hara T, et al. S-1 plus cisplatin versus S-1 alone for first-line treatment of advanced gastric cancer (SPIRITS trial): a phase III trial. Lancet Oncol 2008;9:215-21.

22. He MM, Wu WJ, Wang F, et al. S-1-based chemotherapy versus capecitabine-based chemotherapy as first-line treatment for advanced gastric carcinoma: a meta-analysis. PLoS One 2013;8:e82798.

23. Zhu L, Liu J, Ma S. Fluoropyrimidine-Based Chemotherapy as First-Line Treatment for Advanced Gastric Cancer: a Bayesian Network Meta-Analysis. Pathol 
Oncol Res 2016;22:853-61.

24. Ishigami H, Fujiwara Y, Fukushima R, et al. Phase III Trial Comparing Intraperitoneal and Intravenous Paclitaxel Plus S-1 Versus Cisplatin Plus S-1 in Patients With Gastric Cancer With Peritoneal Metastasis: PHOENIX-GC Trial. J Clin Oncol 2018;36:1922-9.

25. Chan DY, Syn NL, Yap R, et al. Conversion Surgery PostIntraperitoneal Paclitaxel and Systemic Chemotherapy for Gastric Cancer Carcinomatosis Peritonei. Are We Ready? J Gastrointest Surg 2017;21:425-33.

26. Cho H, Ryu MH, Kim KP, et al. Phase I/II study of a combination of capecitabine, cisplatin, and intraperitoneal

Cite this article as: Wang J, Li Z, Qu J, Song N, Chen Y, Cheng Y, Zhang S, Qu X, Liu Y. Clinical outcomes of capecitabine-based versus S-1-based regimens as first-line chemotherapy in patients with unresectable or metastatic gastric cancer: a propensity score matched single-center comparison. J Gastrointest Oncol 2020;11(4):674-684. doi: 10.21037/jgo-20-52 docetaxel (XP ID) in advanced gastric cancer patients with peritoneal metastasis. Gastric Cancer 2017;20:970-7.

27. Lu M, Gao J, Wang XC, et al. Expressions of Thymidylate Synthase, Thymidine Phosphorylase, Class III betatubulin, and Excision Repair Cross-complementing Group 1predict Response in Advanced Gastric Cancer Patients Receiving Capecitabine Plus Paclitaxel or Cisplatin. Chin J Cancer Res 2011;23:288-94.

28. Nakayama Y, Inoue Y, Nagashima N, et al. Expression levels of thymidine phosphorylase (TP) and dihydropyrimidine dehydrogenase (DPD) in patients with gastrointestinal cancer. Anticancer Res 2005;25:3755-61. 
Supplementary

Table S1 Univariate and multivariate analysis of clinical characteristics in relation to overall survival in the observational dataset

\begin{tabular}{|c|c|c|c|c|}
\hline \multirow{2}{*}{ Variables } & \multicolumn{2}{|c|}{ Univariate analysis } & \multicolumn{2}{|c|}{ Multivariate analysis } \\
\hline & $\mathrm{HR}(95 \% \mathrm{Cl})$ & $P$ value & $\mathrm{HR}(95 \% \mathrm{Cl})$ & $P$ value \\
\hline Age & $0.980(0.808-1.190)$ & 0.842 & & \\
\hline \multicolumn{5}{|l|}{$\leq 57$} \\
\hline \multicolumn{5}{|l|}{$>57$} \\
\hline Gender & $1.134(0.919-1.399)$ & 0.240 & & \\
\hline \multicolumn{5}{|l|}{ Male } \\
\hline \multicolumn{5}{|l|}{ Female } \\
\hline ECOG PS & $1.314(0.921-1.874)$ & 0.132 & & \\
\hline \multicolumn{5}{|l|}{0} \\
\hline \multicolumn{5}{|l|}{1} \\
\hline \multicolumn{5}{|l|}{2} \\
\hline Previous gastrectomy & $0.660(0.543-0.802)$ & $<0.001$ & $0.632(0.520-0.769)$ & $<0.001$ \\
\hline \multicolumn{5}{|l|}{ No } \\
\hline \multicolumn{5}{|l|}{ Yes } \\
\hline Location & $0.998(0.905-1.101)$ & 0.972 & & \\
\hline \multicolumn{5}{|l|}{ Cardia/fundus } \\
\hline \multicolumn{5}{|l|}{ Body } \\
\hline \multicolumn{5}{|l|}{ Pylorus/antrum } \\
\hline \multicolumn{5}{|l|}{ Repeat } \\
\hline Differ & $1.126(0.991-1.280)$ & 0.070 & & \\
\hline \multicolumn{5}{|l|}{ Well } \\
\hline \multicolumn{5}{|l|}{ Moderate } \\
\hline \multicolumn{5}{|l|}{ Poor/signet ring } \\
\hline \multicolumn{5}{|l|}{ Unknown } \\
\hline Number of metastasis sites & $1.270(1.130-1.428)$ & $<0.001$ & $1.266(1.130-1.419)$ & $<0.001$ \\
\hline \multicolumn{5}{|l|}{0} \\
\hline \multicolumn{5}{|l|}{1} \\
\hline \multicolumn{5}{|l|}{2} \\
\hline \multicolumn{5}{|l|}{$\geq 3$} \\
\hline Lung metastasis & $1.066(0.664-1.711)$ & 0.791 & & \\
\hline \multicolumn{5}{|l|}{ No } \\
\hline \multicolumn{5}{|l|}{ Yes } \\
\hline Peritoneum metastasis & $1.281(1.012-1.620)$ & 0.039 & & \\
\hline No & & & & \\
\hline Yes & & & & \\
\hline Liver metastasis & $1.198(0.957-1.500)$ & 0.115 & & \\
\hline No & & & & \\
\hline Yes & & & & \\
\hline Bone metastasis & $2.083(1.327-3.272)$ & 0.001 & $1.768(1.116-2.801)$ & 0.015 \\
\hline No & & & & \\
\hline Yes & & & & \\
\hline
\end{tabular}

HR, hazard ratio; Cl, confidence interval; ECOG PS, Eastern Cooperative Oncology Group performance status. 